\title{
Fiber-Optic Raman Spectrum Sensor for Fast Diagnosis of Esophageal Cancer
}

\author{
Jianhua DAI ${ }^{1}$, Xiu $\mathrm{HE}^{2}$, Zhuoyue $\mathrm{LI}^{2}$, Kang $\mathrm{LI}^{2}$, Tingting YANG ${ }^{2}$, Zengling RAN ${ }^{2}$, \\ Lijian YIN ${ }^{1}$, Yao $\mathrm{CHEN}^{1}$, Xiang ZOU ${ }^{3}$, Dianchun $\mathrm{FANG}^{1 *}$, and Guiyong PENG ${ }^{1 *}$ \\ ${ }^{1}$ Institute of Digestive Disease, Southwest Hospital, Army Medical University, Chongqing 40038, China \\ ${ }^{2}$ Fiber Optics Research Center, Key Laboratory of Optical Fiber Sensing \& Communications (Ministry of Education), \\ University of Electronic Science and Technology of China, Chengdu 611731, China \\ ${ }^{3}$ Zolix Instruments Co., Ltd., Beijing 101102, China \\ "Corresponding authors: Daichun FANG and Guiyong PENGＥ-mail: fangdianchun@hotmail.com and pgy63@163.com
}

\begin{abstract}
A fiber-optic Raman spectrum sensor system is used for the fast diagnosis of esophageal cancer during clinical endoscopic examination. The system contains a $785 \mathrm{~nm}$ exciting laser, a Raman fiber-optic probe with 7 large core fibers and a focus lens, and a highly sensitive spectrum meter. The Raman spectrum of the tissue could be obtained within 1 second by using such a system. A signal baseline removal and denoising technology is used to improve the signal quality. A novel signal feature extraction method for differentiating the normal and esophageal cancer tissues is proposed, based on the differences in half-height width (HHW) in $1200 \mathrm{~cm}^{-1}$ to $1400 \mathrm{~cm}^{-1}$ frequency band and the ratios of the spectral integral energy between $1600 \mathrm{~cm}^{-1}-1700 \mathrm{~cm}^{-1}$ and $1500 \mathrm{~cm}^{-1}$ $1600 \mathrm{~cm}^{-1}$ band. It shows a high specificity and effectivity for the diagnosis of esophageal cancer.
\end{abstract}

Keywords: Fiber-optic; Raman spectrum; esophageal cancer

Citation: Jianhua DAI, Xiu HE, Zhuoyue LI, Kang LI, Tingting YANG, Zengling RAN, Lijian YIN, Yao CHEN, Xiang ZOU, Dianchun FANG, and Guiyong PENG, "Fiber-Optic Raman Spectrum Sensor for Fast Diagnosis of Esophageal Cancer," Photonic Sensors, 2019, 9(1): 53-59.

\section{Introduction}

Esophageal cancer is a common cancer of the digestive tumor, and in contrast to many other types of cancer, esophageal cancer is increasing in incidence and mortality [1]. Globally, an estimated 450000 new cases and 400000 deaths occurred in 2012. Over the past 40 years, the incidence of esophageal cancer has increased nearly $50 \%$, largely attributed to a dramatic increase in the incidence of esophageal adenocarcinoma [2]. Given the high fatality rate of all types of esophageal carcinomas and the rapidly increasing incidence of esophageal adenocarcinoma, the identification of high-risk lesions together with therapeutic interventions is the most critical measure to improve survival rates of esophageal cancer patients [3]. However, the conventional diagnosis methods [4-10] are mainly based on endoscope at present, which heavily relies on visual assessment of structural and morphological changes of the tissue surface, resulting in a poor diagnostic accuracy. In addition, the final correct diagnosis for the endoscope method is time-consuming because it needs pathologic

Received: 13 August 2018 / Revised: 2 September 2018

(C) The Author(s) 2018. This article is published with open access at Springerlink.com

DOI: $10.1007 / \mathrm{s} 13320-018-0516-7$

Article type: Regular 
examination with tissue biopsies. Therefore, taking into account of the enormous rise in incidence rates of esophageal cancer and the existing clinical challenges, the need of new advanced diagnosis method has never been greater. Recent attention has thus been directed toward biological detection using optical spectroscopy and imaging, such as surface plasmon resonance spectroscopy [11] and Raman scattering detection [12-16]. Among these, Raman spectroscopy represents a unique optical vibrational technique based on the fundamental premise of inelastic light scatting for tissue diagnosis and characterization [12-16]. In the last 2 decades, there has been accumulating evidences on the accurate diagnostic capability of Raman spectroscopy through comprehensive in vitro studies. As to quick Raman endoscopic applications, however, they have been limited not only by the difficulty in capturing the inherently very weak tissue Raman signals [17, 18], but also by the slow speed of spectral measurements ( $>5 \mathrm{~s})[15,16]$. In addition, the conventional methods to differentiate normal and pathological tissues based on the differences of peak wavelength and relative intensity [12-18] can not provide satisfied diagnosis specificity for clinical applications. To tackle these challenges, by the combination of the high-performance fiber-optic Raman spectrum sensor system and signal baseline removal and denoising technology, the Raman spectrum of the tissue is obtained within 1 second with high quality. In order to differentiate normal and esophageal cancer tissues effectively, a novel method based on tracking the half-height width (HHW) of Raman peaks and ratios of integral energy at different bands is utilized.

\section{Experimental system and the analysis method}

A schematic and relative physical device diagram of the fiber-optic Raman spectrum sensing system for fast diagnosis of esophageal cancer is shown in Figs. 1 and 2, respectively. Briefly, the system consists of a near-infrared semiconductor laser with a wavelength $\lambda_{\mathrm{ex}}=785 \mathrm{~nm}$, an imaging spectroscopy equipped with a high-performance charge coupled device (CCD), a specially designed $2 \mathrm{~mm}$ (outer diameter) fiber-optic Raman probe for both in laser light delivery and in tissue Raman signal collection, and a super-efficiency computer for signal processing. Figure 1 also displays the zoom of the fiber-optic Raman probe, which is composed of a laser input fiber of $272 \mu \mathrm{m}$ in core diameter and 7 collective fibers of $300 \mu \mathrm{m}$ in core diameter, and a miniature $1.0 \mathrm{~mm}$ focus lens is coupled to the fiber tip of the Raman probe to tightly focus the excitation light onto the tissue, enabling the effective Raman spectrum collection from the tissue. In this system, the fiber-optic probe is simultaneously connected to the near-infrared semiconductor laser and the imaging spectrograph with the $\mathrm{CCD}$, and the imaging spectrometer is connected to the computer to visualize the Raman spectra timely. Therefore, we firstly obtained a piece of tissue sample with an endoscope and then placed the fiber-optic Raman probe in gentle contact with the tissue surface for tissue characterization and fast diagnosis.

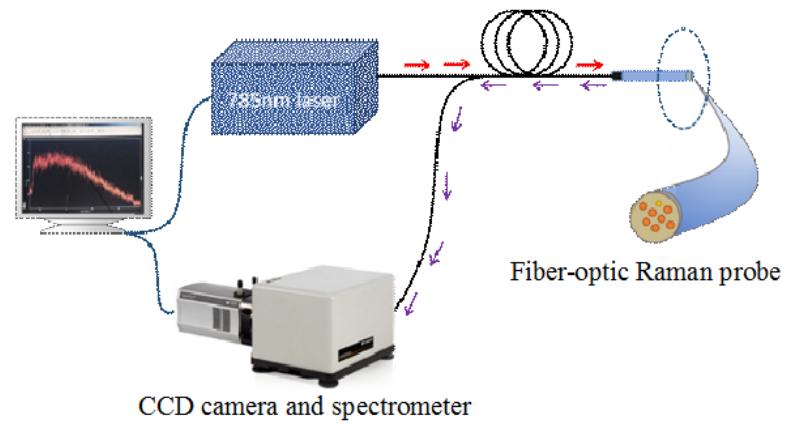

Fig. 1 A schematic of the fiber-optic Raman spectrum sensing system for the fast diagnosis of esophageal cancer. 


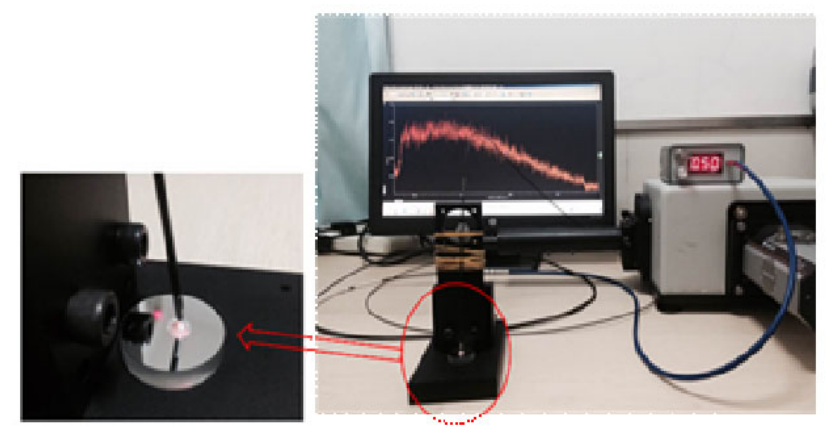

Fig. 2 Physical device diagram of the fiber-optic Raman spectrum sensing system for the fast diagnosis of esophageal cancer.

In the experiment, the low signal-to-background ratio spectra from human esophageal cancer tissues are obtained by the Raman spectroscopy without any sample preparation. The typical original Raman spectra of normal tissues in the range of $800 \mathrm{~cm}^{-1}$ $-1800 \mathrm{~cm}^{-1}$ are shown in Fig. 3(a). Figure 3(a) indicates that the spectrum is affected by noise and a strong sloping fluorescence background. It is very difficult to extract the qualitative or quantitative feature due to these interferences. To obtain the high signal-to-background ratio and characteristic Raman spectra, we adopt one background elimination method consisting of two parts: baseline removal and smoothing based on the fast Fourier transform (FFT). All calculations are carried out with the LABVIEW software. Firstly, the original spectral data are preprocessed by removing the baseline approach. The calculation result shown in Fig. 3(b) indicates that the strong sloping fluorescence background is obviously eliminated and a crowd of sharp peaks are emerged. And then, by smoothing processing of a spectrum using the FFT, the peaks in the processed spectrum as shown in Fig. 3(c) become smoothing with a high signal-to-background ratio. All these preprocessing are completed within $1 \mathrm{~s}$, and the processed results can be displayed on the computer screen in time.

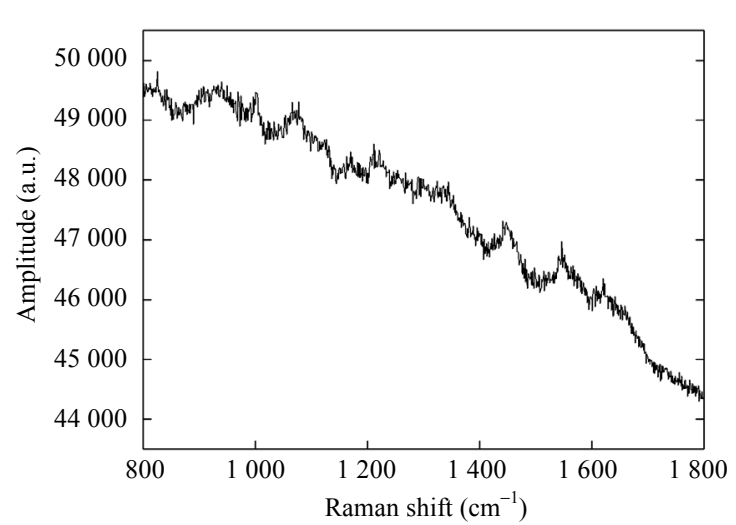

(a)

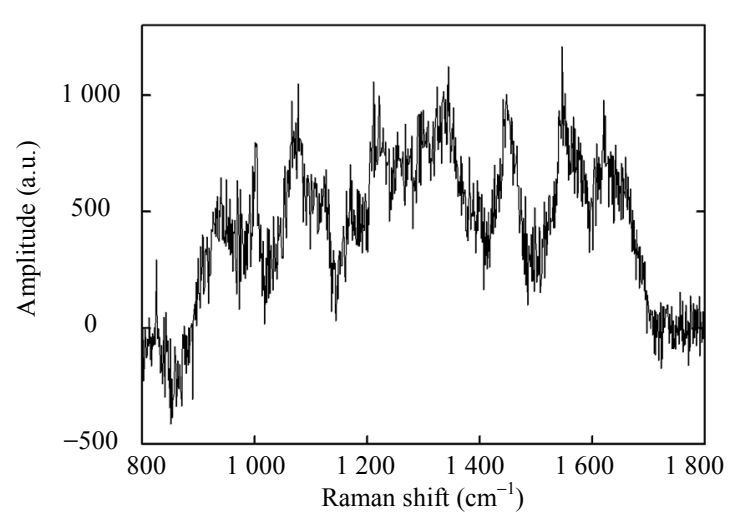

(b)

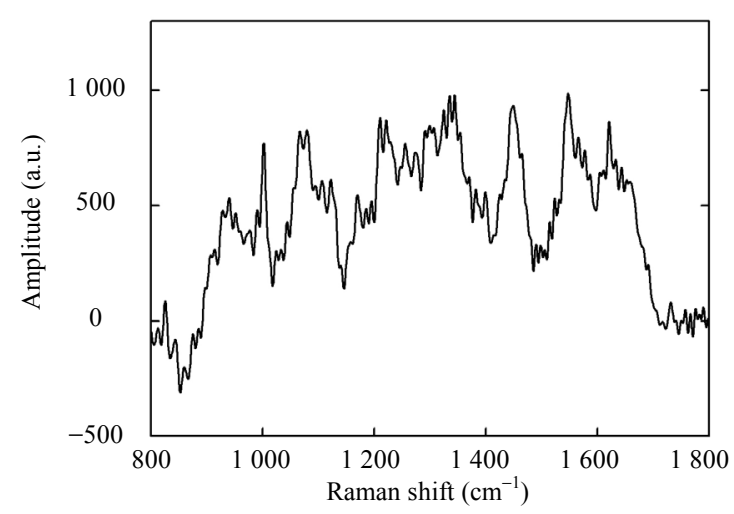

(c)

Fig. 3 Data processing: (a) the original Raman spectrum of tissue in the range of $800 \mathrm{~cm}^{-1}-1800 \mathrm{~cm}^{-1}$, (b) the baseline removal Raman spectrum, and (c) the last Raman spectrum after smoothing.

\section{Results and discussion}

We test four types of esophageal cancers which are poorly differentiated carcinoma, squamous cell carcinoma, adenocarcinoma, and carcinoma in situ, respectively. Each specimen from four different human vivo tissues, including 2 normal and 
2 malignant tissues, is obtained after informed consent. Figures 4(a) - 4(d) show the Raman spectra of poorly differentiated carcinoma and normal tissues, squamous cell carcinoma and normal tissues, adenocarcinoma and normal tissues, carcinoma in situ and normal tissues, respectively. In each figure, each spectrum is the average spectrum of the same types of tissues. There exist two evident differences between the carcinoma and normal tissues spectra. As to the first one (in the solid line box), compared with the normal tissues spectrum, the carcinoma tissues spectrum has a broader peak spectrum half height width in the range of $1100 \mathrm{~cm}^{-1}$ $-1400 \mathrm{~cm}^{-1}$. As to the second one (in the dashed box), ranging from $1500 \mathrm{~cm}^{-1}$ to $1600 \mathrm{~cm}^{-1}$, the spectral integral energies of carcinoma tissues are higher than those of the normal tissues spectra, consequently resulting in a lower integral energy ratio between $1600 \mathrm{~cm}^{-1}-1700 \mathrm{~cm}^{-1}$ and $1500 \mathrm{~cm}^{-1}$ $1600 \mathrm{~cm}^{-1}$. Additionally, we do a quantitative

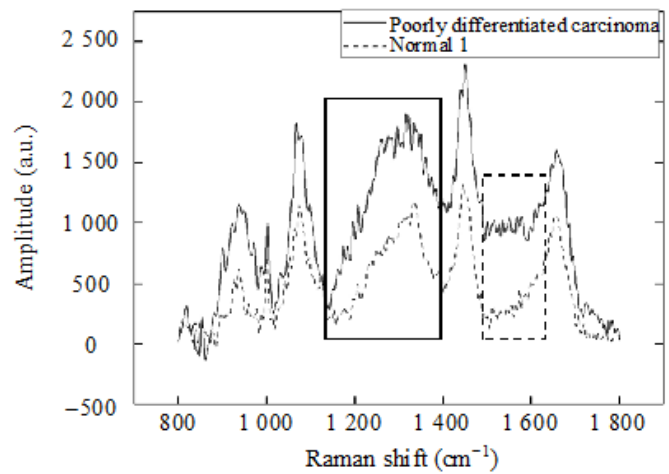

(a)

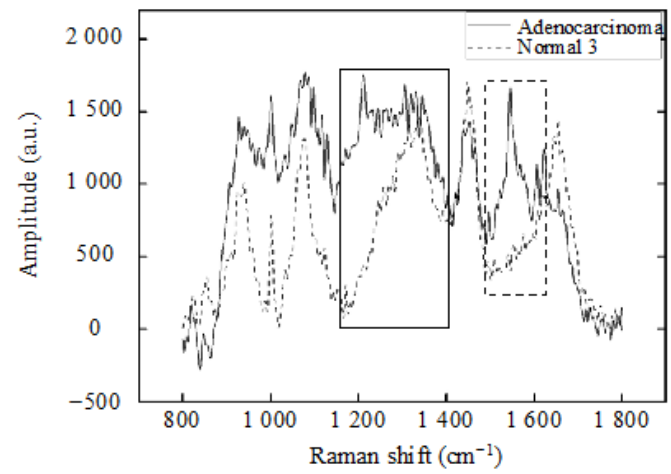

(c) analysis about two differences as shown in Tables 1 and 2 in detail, respectively. Table 1 gives the HHWs of the Raman spectra of all specimens in range of $1200 \mathrm{~cm}^{-1}-1400 \mathrm{~cm}^{-1}$, and their distribution diagrams are shown in Fig. 5. Table 1 and Fig. 5 show that the HHW of each carcinoma spectrum is nearly as twice as that of the normal tissue, in the range of $1200 \mathrm{~cm}^{-1}-1400 \mathrm{~cm}^{-1}$. Table 2 and Fig. 6 provide the ratios of the spectral integral energies between $1600 \mathrm{~cm}^{-1}$ to $1700 \mathrm{~cm}^{-1}$ and $1500 \mathrm{~cm}^{-1}$ to $1600 \mathrm{~cm}^{-1}$, respectively. The ratios of the carcinoma tissue samples are around 1, and the ratios of normal tissue samples are approximated 2 . There is 2-time difference between them. Raman spectra signals are attributed to biological compounds such as proteins, lipids and DNA as well as to chemical bonds within these compounds such as $\mathrm{C}=\mathrm{O}$ stretch in amide $\mathrm{I}\left(1645 \mathrm{~cm}^{-1}-1680 \mathrm{~cm}^{-1}\right)$ and $\mathrm{N}-\mathrm{H}$ bending in amide III $\left(1230 \mathrm{~cm}^{-1}-1310 \mathrm{~cm}^{-1}\right)$ [19]. The differences indicate that the esophageal

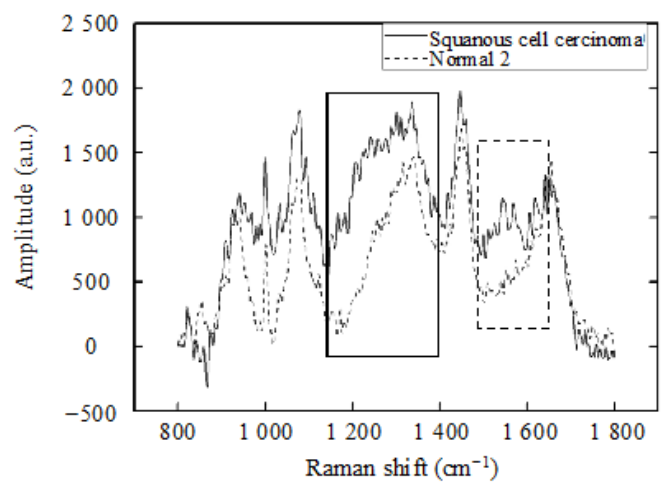

(b)

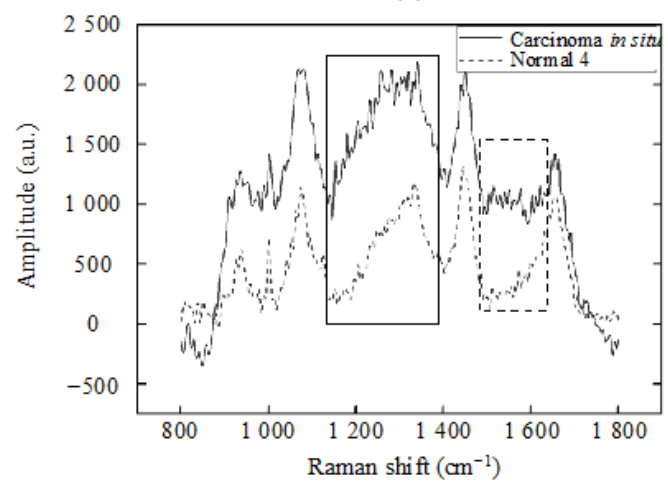

(d)

Fig. 4 Raman spectra of (a) the poorly differentiated carcinoma and normal tissues, (b) squamous cell carcinoma and normal tissues, (c) adenocarcinoma and normal tissues, and (d) carcinoma in situ and normal tissues. 
cancer tissues exist in some unusual and new proteins induced by DNA mutation compared with the normal tissues. Therefore, the combination of the fiber-optic Raman sensor and optimization signal analysis method can realize the fast diagnosis of esophageal cancers.

Table 1 Peak spectrum HHW of all specimens in a range of $1200 \mathrm{~cm}^{-1}-1400 \mathrm{~cm}^{-1}$.

\begin{tabular}{ccc}
\hline Sample code & $\begin{array}{c}\text { Effective peak locations } \\
\left(\mathrm{cm}^{-1}\right)\end{array}$ & $\begin{array}{c}\text { HHWs } \\
\left(\mathrm{cm}^{-1}\right)\end{array}$ \\
\hline PDC1 & 1315.843 & 114.59 \\
PDC1 & 1298.3 & 155.703 \\
SCC1 & 1263.979 & 178.824 \\
SCC2 & 1336.364 & 148.506 \\
AC1 & 1210.24 & 201.321 \\
AC2 & 1343.517 & 152.849 \\
CIS1 & 1340.453 & 140.687 \\
CIS2 & 1263.934 & 170.451 \\
N1-1 & 1340.453 & 70.898 \\
N1-2 & 1320.153 & 69.151 \\
N2-1 & 1333.294 & 86.594 \\
N2-2 & 1340.294 & 82.691 \\
N3-1 & 1332.195 & 84.763 \\
N3-2 & 1343.189 & 83.468 \\
N4-1 & 1342.361 & 72.904 \\
N4-2 & 1335.456 & 70.216 \\
\hline
\end{tabular}

"PDC" denotes poorly differentiated carcinoma tissue sample, "SCC" denotes squamous cell carcinoma tissue sample, "AC" denotes adenocarcinoma tissue sample, and "CIS" denotes carcinoma in situ tissue sample. "N" denotes the normal tissue sample. "N1-" denotes the normal tissue sample compared with the poorly differentiated carcinoma tissue sample, "N2-" denotes the normal tissue sample compared with squamous cell carcinoma tissue sample, "N3-" denotes the normal tissue sample compared with the adenocarcinoma tissue sample, and "N4-" denotes the normal tissue sample compared with the carcinoma in situ tissue sample.

Table 2 Ratios of the integral energies of two Raman spectrum bands.

\begin{tabular}{cccc}
\hline Sample code & E1 (a.u.) & E2 (a.u.) & E2/E1 \\
\hline PDC1 & 1065818 & 127835.7 & 1.27 \\
PDC1 & 57768.26 & 56235.55 & 0.97 \\
SCC1 & 95928.18 & 103230.9 & 1.08 \\
SCC2 & 113420 & 110552.9 & 0.97 \\
AC1 & 63960.95 & 55657.35 & 0.87 \\
AC2 & 106813 & 84281.22 & 0.79 \\
CIS1 & 105774.4 & 112583.4 & 1.06 \\
CIS2 & 77833.18 & 77125.31 & 0.99 \\
N1-1 & 52473.05 & 102472.7 & 1.95 \\
N1-2 & 30702.71 & 74365.92 & 2.42 \\
N2-1 & 46235.12 & 92156.45 & 1.99 \\
N2-2 & 57368.78 & 124598.46 & 2.17 \\
N3-1 & 35248.49 & 69851.47 & 1.98 \\
N3-2 & 44256.46 & 90124.32 & 2.04 \\
N4-1 & 39541.48 & 79513.54 & 2.01 \\
N4-2 & 40694.56 & 82145.54 & 2.02 \\
\hline
\end{tabular}

"E1" denotes the integral energy from $1500 \mathrm{~cm}^{-1}$ to $1600 \mathrm{~cm}^{-1}$, and "E2" denotes the integral energy from $1600 \mathrm{~cm}^{-1}$ to $1700 \mathrm{~cm}^{-1}$.

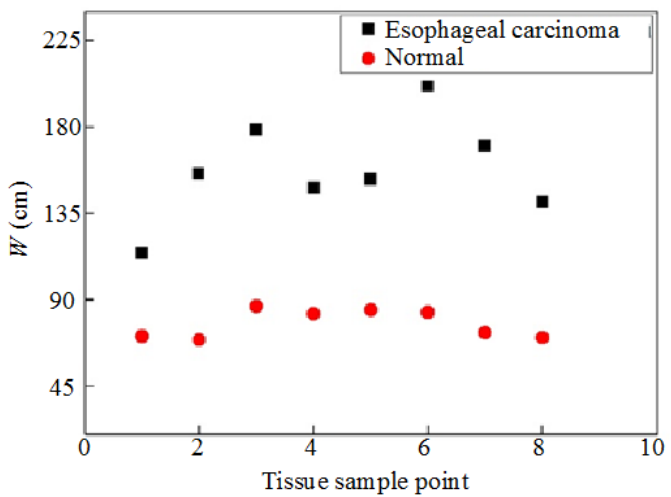

Fig. 5 Peak spectrum HHW distribution diagram of the Raman spectrum of normal and carcinoma tissues in the range of $1100 \mathrm{~cm}^{-1}-1400 \mathrm{~cm}^{-1}$.

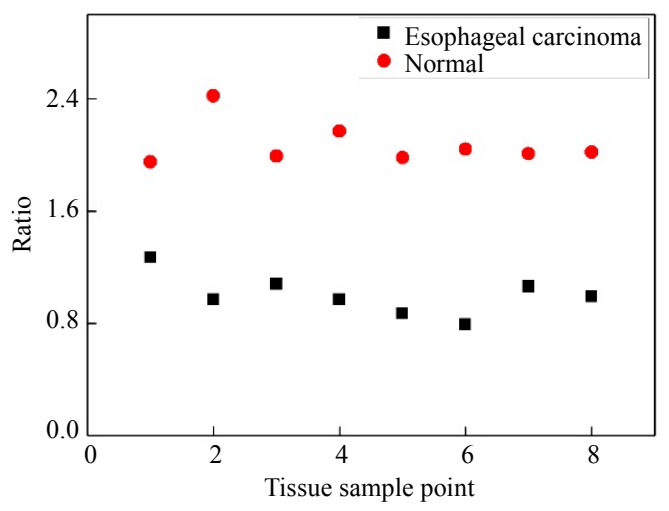

Fig. 6 Ratio distribution diagram of the integral energy of two Raman spectrum bands of the normal and carcinoma tissues.

\section{Conclusions}

A high-performance fiber-optic Raman spectrum sensing system and effective signal process/analysis method are proposed and demonstrated for the diagnosis of esophageal cancers. The Raman spectrum of the tissues is obtained within 1 second by the combination of the high-performance fiber-optic Raman spectrum sensing system and signal filtering processing technology. The proposed analysis method based on HHWs and integral energy ratios can differentiate the normal and pathological tissues effectively. It is expected that the method proposed can be used to solve the problem of clinical fast diagnosis of esophageal cancer with the good accuracy.

\section{Acknowledgment}

This word was supported by multicenter clinical 
study of endoscopic diagnosis of early esophageal cancer (Grant No. SWH2016ZDCX3007); the State 111 Project (Grant No. B14039); Application of endoscopic minimally invasive technique in diagnosis and treatment of digestive tract injury and disease (Grant No. SWH2016ZDCX2011); Study of effect on fibroblast transdifferentiation and prevention of postoperative esophagus stenosis through regulating the TRADD (Grant No. 81470907); the National Natural Science Foundation of China (NSFC) (Grant Nos. 51627806 and 51875091); Application of Raman imaging in the diagnosis of gastric cancer (Grant No. cstc2015shmszx10017).

Open Access This article is distributed under the terms of the Creative Commons Attribution 4.0 International License (http://creativecommons.org/licenses/by/4.0/), which permits unrestricted use, distribution, and reproduction in any medium, provided you give appropriate credit to the original author(s) and the source, provide a link to the Creative Commons license, and indicate if changes were made.

\section{References}

[1] A. Jemal, R. Siegel, J. Q. Xu, and E. Ward, "Cancer statistics," CA: A Cancer Journal for Clinicians, 2010, 60(5): 277-300.

[2] L. M. Brown, S. S. Devesa, and W. H. Chow, "Incidence of adenocarcinoma of the esophagus among white Americans by sex, stage, and age," Journal of the National Cancer Institute, 2008, 100(16): 1184-1187.

[3] S. S. Devesa, W. J. Blot, and J. F. Fraumeni, "Changing patterns in the incidence of esophageal and gastric carcinoma in the United States," Cancer, 1998, 83(10): 2049-2053.

[4] R. Krishnamoorthi, S. Singh, K. Ragunathan, D. A. Katzka, K. K. Wang, and P. G. Iyer, "Risk of recurrence of Barrett's esophagus after successful endoscopic therapy," Gastrointestinal Endoscopy, 2016, 83(6): 1090-1106.

[5] T. Oyama, A. Tomori, K. Hotta, S. Morita, K. Kominato, M. Tanaka, et al., "Endoscopic submucosal dissection of early esophageal cancer," Clinical Gastroenterology and Hepatology, 2005, 3(7): S67-S70.

[6] M. Fujishiro, N. Yahagi, N. Kakushima, S. Kodashima, Y. Muraki, S. Ono, et al., "Endoscopic submucosal dissection of esophageal squamous cell neoplasms," Clinical Gastroenterology and Hepatology, 2006, 4(6): 688-694.

[7] T. Ohki, M. Yamato, M. Ota, R. Takagi, D. Murakami, M. Kondo, et al., "Prevention of esophageal stricture after endoscopic submucosal dissection using tissue-engineered cell sheets," Gastroenterology, 2012, 143(3): 582-588.

[8] T. Mizumoto, T. Hiyama, S. Ok, N. Yorita, K. Kuroki, M. Kurihara, et al., "Curative criteria after endoscopic resection for superficial esophageal squamous cell carcinomas," Digestive Diseases and Sciences, 2018, 63(6): 1605-1612.

[9] C. Fleichmann and H. Messmann, "Endoscopic treatment of early esophageal squamous neoplasia," Minerva Chirurgica, 2018, 73(4): 378-384.

[10] L. Sreedharan, G. C. Mayne, D. I. Watson, T. Bright, R. V. Lord, A. Ansar, et al., "MicroRNA profile in neosquamous esophageal mucosa following ablation of Barrett's esophagus," World Journal of Gastroenterology, 2017, 23(30): 55085518.

[11] S. N. Choudhury, B. Konwar, S. Kaur, R. Doley, and B. Mondal, "Study on snake venom protein-antibody interaction by surface plasmon resonance spectroscopy," Photonic Sensors, 2018, 8(3): 193-202.

[12] C. Xiao, Z. B. Chen, M. Z. Qing, D. X. Zhang, and L. Fan, "Composite sinusoidal nanograting with long-range SERS effect for label-free TNT detection," Photonic Sensors, 2018, 8(3): 1-11.

[13] S. S. Cui, S. Zhang, and S. H. Yue, "Raman spectroscopy and imaging for cancer diagnosis," Journal of Healthcare Engineering, 2018: 8619342-1-8619342-11.

[14] T. D. Wang, G. Triadafilopoulos, J. M. Crawford, L. R. Dixon, T. Bhandari, P. Sahbaie, et al., "Detection of endogenous biomolecules in Barrett's esophagus by Fourier transform infrared spectroscopy," Proceedings of the National Academy of Sciences, 2007, 104(40): 15864-15869.

[15] M. G. Shim, W. K. S L. Michel, N. E. Marcon, and B. C. Wilson, "In vivo near-infrared Raman spectroscopy: demonstration of feasibility during clinical gastrointestinal endoscopy," Photochemistry and Photobiology, 2000, 72(1): 146-150.

[16] Z. W. Huang, S. K. Teh, W. Zheng, J. H. Mo, K. Lin, X. Z. Shao, et al., "Integrated Raman spectroscopy and trimodal wide-field imaging techniques for real-time in vivo tissue Raman measurements at endoscopy," Optics Letters, 2009, 34(6): 758760 .

[17] G. Shetty, C. Kendall, N. Shepherd, N. Stone, and H. Barr, "Raman spectroscopy: elucidation of biochemical changes in carcinogenesis of oesophagus," British Journal of Cancer, 2006, 94: 1460-1464. 
[18] M. S. Bergholt, W. Zheng, K. Y. Ho, M.Teh, K. G. Yeoh, J. B. Y. So, et al., "Fiber-optic confocal Raman spectroscopy for real-time in vivo diagnosis of dysplasia in Barrett's esophagus," Gastroenterology, 2014, 146(1): 27-32.

[19] Y. G. Hu, A. G. Shen, T. Jiang, Y. Ai, and J. M. Hu,
"Classification of normal and malignant human gastric mucosa tissue with confocal Raman microspectroscopy and wavelet analysis," Spectrochimica Acta Part A: Molecular and Biomolecular Spectroscopy, 2008, 69(2): 378-382. 